EPJ Web of Conferences 95,02001 (2015)

DOI: 10.1051/epjconf/20159502001

(C) Owned by the authors, published by EDP Sciences, 2015

\title{
Engaging young people with our science
}

\author{
Marjorie G. Bardeen ${ }^{1}$ \\ ${ }^{1}$ Fermi National Accelerator Laboratory, Batavia, IL 60510 USA
}

\begin{abstract}
Communication, education and outreach are increasingly important elements of the particle physics research agenda as acknowledged in recent European Strategy and U.S. Community Summer Study reports. These efforts help develop the next generation of researchers and a scientifically literate citizenry. We describe some examples that engage young people with our science.
\end{abstract}

\section{Introduction}

We reach out to young people to inspire our next generation workforce and to convince next generation voters that our science is exciting and worthy of their support. It's not so difficult to make outreach part of our research agenda. We need to know our audience, personalize our science with stories, engage youngsters with interactive presentations and activities to bring them into our world.

\section{Creating interest for the youngest children}

When we reach out to the youngest children, our goal is to create interest rather than teach physics. We go where they are: in schools, libraries, museums, where people meet one another-at shopping malls, festivals, and markets. We share our work by engaging children in conversation and appropriate hands-on activities. We lead by asking questions and getting children to ask questions too. This is often a different way of communicating than we are used to. However, many available resources make this easier. The International Particle Physics Outreach Group (IPPOG) has an excellent online resources database [1].

\subsection{Chania science expo}

We had just such an event, Science Hoists Sails, during the conference. Held at the Chania Sailing Club, Neorio Moro, we organized an ALTAS Masterclass and set up seven activities that gave visitors a chance to explore particle structure, paths and patterns, conservation laws, collect data to make a measurement using indirect evidence, and put together a jigsaw puzzle of CERN's ATLAS detector. Several conference participants and accompanying persons served as explainers to engage the visitors with these hands-on activities meant to interest people ages 9 to 90.

The context for the activities was techniques particle physics uses to make discoveries.

\section{The Standard Model organizes what we know about subatomic particles.}

1. Quark Workbench: Using puzzle pieces to look for patterns in how these pieces can fit together, 
building baryons and mesons from quarks. The puzzle pieces obey, as much as possible, the Standard Model rules limiting the quark composition of bound states.

2. What's Inside? Probing clay balls to determine internal structure to find the "nucleus," an object such as a coin and describe its size, shape, and location.

Magnetic and electric fields accelerate, bend and focus beams of charged particles.

3. Detector Slice: Seeing how different particles interact with the CMS detector and can be identified by the patterns they make.

4. Checking out the symmetry and complexity of ATLAS by assembling a jigsaw puzzle.

Event displays visualize data. Application of conservation of momentum and energy reveals new phenomena.

5. Higgs Signature: Identifying Higgs signatures in an event display game.

6. Energy \& Momentum: Colliding ball bearings on a track to study conservation of energy and momentum.

Indirect evidence provides data to study particles too small and fleeting to see.

$\leftarrow 7$. Rolling with Rutherford: Using statistics to make an indirect measurement of the size of an object, just as physicists do when they use accelerators to measure properties of very small particles.

Histograms represent data for analysis and interpretation.

$\leftarrow 7$. Rolling with Rutherford: Creating and interpreting a histogram of the number of hits on the target.

\subsection{Presentations}

Perhaps the easiest form of outreach is to give a presentation for a group of children at school or a museum or for a program such as scouting. We can put on an exciting "participatory" presentation showcasing physics topics. By participating in activities and demonstrations, students become engaged in developing a deeper understanding of concepts such as force and motion, electricity and magnetism, light and color. One can create an entertaining assembly-style cryogenics show. For example, Fermilab's Mr. Freeze is booked months in advance! Or take the show on the road. By outfitting a van, such as the one at the University of Illinois, Urbana-Champaign, undergraduates can take a demonstration show to area schools. Students show young people that science is fun and that scientists are pretty normal people. Tips for successful presentations:

1. Introduce yourself. Let the children know something about you. Find out something about them.

2. Stimulate thinking by asking questions.

3. Let children see for themselves-learn by doing.

\subsection{Informal classes and camps}

Science is a social enterprise; science learning should be social too. The more experiences children have working with others on a engaging project, the sooner they will begin to appreciate the wonderful world of science and the adventures it offers. You could develop and offer an informal science class for a museum or community program. A class can turn into a scout badge workshop if based on topics aligned with scout programs. Typically, classes last a half or full day. However, stringing several classes together can become a one-week science camp. Tips for successful classes:

1. Begin with an objective e.g., Gain skills and knowledge to successfully build and test a long flying airplane.

2. Keep to one main idea per class so children explore in depth.

3. Mirror the way scientists approach and solve problems: use a variety of methods to investigate phenomena and help children use evidence and reasoning to support claims they make or design an object/instrument they test. 


\section{Non-formal and informal learning for student groups}

Unlike formal learning, non-formal learning takes place outside the school curriculum. Structured learning situations aim to increase learner's knowledge and skills but without the rigor of syllabi or tests. Examples from our colleagues include labs at DESY Zeuthen and the Stockholm House of Science and Fermilab's Saturday Morning Physics (SMP). Particle Physics Cascades, competitions at the University of Birmingham in the UK, is an example of informal where learning is not intentional from the learner's point of view and there are no set learner outcomes.

\subsection{School labs}

To make modern science accessible, staff members at the Stockholm House of Science [2], a university science laboratory, guide students of all ages and their teachers through various ageappropriate, hands-on activities. The goals are to increase interest and knowledge in science, technology, engineering, and mathematics (STEM), to strengthen contacts between schools and the university, and to show that university studies can lead to interesting jobs. Many of the projects are not easy to perform in schools and are unique to the House of Science, located on the AlbaNova University campus and at the Bergianska Tradgarden botanic garden. Staff, including Ph.D. and undergraduate students, offers programs in biology, chemistry, physics, mathematics and technology. Programs can be part of a school course, just for inspiration or for teacher professional development. Teacher workshops cover a variety of topics, for example, Being Mathematical builds on the school program, "Natural Mathematics."

A focus of the school lab in DESY Zeuthen [3] is the vacuum lab where staff offers a half-day program for students in grades 4-8 and a full-day program for students in grades 9-10. Students work in small groups on one or more of twelve experiments that require a vacuum. Students have access to diaphragm and rotary vane pumps not readily available in schools. For example, in the Hemispheres of Magdeburg, students try to pull apart two hemispheres that can be separated only with immense effort demonstrating how strong the air pressure is. In another room, high school students conduct experiments with cosmic particles. They use modern measurement and analysis methods to answer questions and enhance their skills presenting results. Staff also offers workshops for primary school teachers with simple experiments to illustrate phenomena associated with the topics of air pressure and vacuum. For high school teachers, DESY offers workshops on experiments that measure cosmic particles. Netzwerk Teilchenwelt makes the experiments available to teachers.

\subsection{Saturday morning science}

One of 15 active Saturday science programs in Canada, Germany and the United States, Fermilab's Saturday Morning Physics [4] is a free series of nine lectures and tours offered to further understanding and appreciation of modern physics among high school students. The program design emphasizes the opportunity to "kindle a lifelong curiosity" rather than "teach" particle physics. Volunteer physicists give the lectures, and postdocs and grad students lead tours. Session topics include the usual suspects-accelerators, detectors, cosmology, and theory of relativity-but also topics of interest to the students, such as energy and climate and physics and society. Physics departments have an opportunity to offer a wider program with lectures on current research areas. At the University of Illinois, the current Saturday Physics for Everyone [5] include Building Mountains, When Stars Attack, and How Cells Sense Their Environment.

\subsection{Video competition}


The School of Physics and Astronomy at the University of Birmingham, UK, organizes particle physics competitions to encourage A level and GCSE students to share their enthusiasm for physics with other students in their own school. Called Cascades of Particle Physics [6], schools from throughout the UK enter teams of students who prepare either a live presentation which they film, Cascade Live, or a video, Cascade Video, to introduce some concepts of particle physics in innovative and entertaining ways. Using well-defined criteria, a panel composed of a particle physicist, teacher, Ph.D. student, and non-specialist judges the entries. A handful of finalists come to campus to give their live presentations or show their videos. The visit includes a short lecture and tour, light lunch, an awards ceremony in which students receive certificates, and schools may receive prizes. (Monetary prizes depend on sponsorship from outside sources.) As always, the day includes plenty of time for students to meet with fellow competitors and members of the particle physics group. This competition is relatively easy to organize because the students do most of the work at school.

\section{Hands-on analysis with real data}

Particle physicists have made experimental data available for high school students at least since the time of CERN's Large Electron-Positron Collider and the discovery of the top quark at Fermilab. We gained experience engaging students in making measurements with the data so they get a broader frame of reference for science than they normally get in school. They begin to understand how scientists make discoveries and talk about their work. The following examples build from a one-day event called masterclass to school-based guided investigations and cosmic ray studies to summer internships with university and lab research groups.

\subsection{International Masterclass}

The International Particle Physics Outreach Group (IPPOG) [7] offers International Masterclasses [8] for the ATLAS, ALICE, CMS and LHCb experiments at CERN. High school students go to a research center near their home to become scientists for a day. Lectures from active scientists give insight in topics and methods of basic particle physics research. After these introductory lectures, students learn how to examine large samples of recent data, mastering real event-display programs, software tools and analysis methods. Students perform one of six measurements on real data with help from mentor physicists. They use event displays to build up statistics through event-by-event analysis. In ATLAS and CMS masterclasses, students study (mostly) leptonic decays to determine lepton ID (electron, muon) and candidate particle ID (J/Psi, Upsilon, W, Z, Higgs). Students create mass plots to find the mass of the $\mathrm{Z}$ boson and a possible Higgs signal and other peaks in the mass plot to reveal additional particles. In ALICE, students look for strange matter and the effect of the quark-gluon plasma on particle production. In $\mathrm{LHCb}$, they look for displaced vertices in B decays.

Students combine the results of their analyses with those of their peers to draw conclusions based on statistics that they could not make working alone with a relatively small number of events. The keys to a successful measurement are interaction with physicists and a robust group discussion to conclude the analysis. In addition, students may have lunch with physicists and go on a short tour. At the end of the day, they join a videoconference for discussion and combination of their results with students at other sites just as particle physics collaborations do.

Surveys in Europe and the United States indicate that students liked the masterclass " very miuch » or "much». Evaluative data from U.S. masterclasses found that students who received as little as three hours preparation learned more and reported higher rates of satisfactions than students who came unprepared. For some students lectures were a highlight. Others liked the hands-on measurements.

\subsection{Guided student investigations}

For students ready to perform authentic research, three QuarkNet [9] e-Labs offer studies based on 
data from either CMS, the Laser Interferometer Gravitational Wave Observatory (LIGO) or classroom cosmic ray detectors. Students make correlations, transform provided data into usable form, and calibrate or determine useful data. Students draw conclusions supported by evidence and provide reasoning.

e-Labs, delivered as Web-based portals accessible in the classroom and at home, are implemented with the ever-expanding capabilities of Web-based media. e-Labs explore the potential of using the Internet and distributed computing in high school classes and provide an opportunity for students to:

- Organize and conduct authentic research.

- Experience the environment of scientific collaborations.

- Possibly make real contributions to a burgeoning scientific field.

Studies are problem-based, student driven and technology dependent. Students reach beyond classroom walls to explore data with other students and experts and share results, publishing work to a worldwide audience. Students can discover and extend the research of other students, modeling the processes of modern, large-scale research projects.

From start to finish e-Labs are student-led, teacher-guided projects. Students need only a Web browser to access a project map with milestones that allows students to set the research plan rather than follow a step-by-step process common in other online projects. Most importantly, e-Labs build the learning experience around the students' own questions and let them use the very tools that scientists use.

Students contribute to and access shared data, most derived from professional research databases. They use common analysis tools, store their work and use metadata to discover, replicate and confirm the research of others. Teaching tools such as student and teacher logbooks, pre- and post-tests and an assessment rubric aligned with learner outcomes help teachers guide student work. Constraints on interface designs and administrative tools such as registration databases give teachers the "one-stop-shopping" they seek for multiple e-Labs. Teaching and administrative tools also allow staff to track usage and assess the impact on student learning. [10]

\subsection{Cosmic ray studies}

As early as 1994, physicists began exploring the possibility of studying cosmic rays by building sparse detector arrays where the detectors were located at schools, usually on rooftops. For the most part, custom-built electronics controlled the detectors, allowing precision measurement of timing and energy as well as time synchronization across sites. Data went directly to the research group for analysis. Support staff was essential to keep the system operating. Projects offered workshops where teachers learned about the physics of cosmic rays, assembled instruments, and in some cases practiced calibrating detectors, and collected and analysed data. Students may have participated with their teachers in the workshop to develop a team of local experts. These workshops were essential for successful classroom implementation. Mastery came only later after teachers had several years of experience working with the detector. Many of these projects have concluded, but several are ongoing in Germany, the Netherlands, the United Kingdom, and the United States.

\subsubsection{QuarkNet}

QuarkNet provides detector kits-DAQ board, counters, Global Positioning System (GPS) and various cables - to teachers and their students. Users build a classroom detector, learning about the components and how they work together to collect data. They also learn how to make their data available to users around the world. At school they can set up their detector as a telescope to do flux or muon lifetime studies or as an array to do shower studies. They can upload their data to the Cosmic 
Ray e-Lab (see 4.2) where they find data from across the collaboration and analysis and reporting tools. Key to the QuarkNet program is the ability of students to design and carry out their own investigation with back-up support from teachers and staff as needed. As of November 1, 2014, the collaboration has uploaded over 62,000 data files from 600 schools in 62 states and countries, each file representing one day of data. Users have saved over 16,000 analysis plots. The QuarkNet DAQ board necessary for a compatible data format is found in detectors in the United States and 23 other countries. [11]

\subsubsection{HISPARC}

The High School Project on Astroparticle Research with Cosmics (HiSPARC) [12] represents the most common approach to student cosmic ray research. HiSPARC offers schools the opportunity to participate in serious astrophysics research. The purpose is to conduct fundamental research and provide an opportunity for younger students to participate in the research. Detectors, linked to GPS satellites and university research centers, are deployed on school and college rooftops. Typically, detectors spaced meters apart are placed in weatherproof rooftop boxes with a computer for local data acquisi tion and storage. Students maintain and operate the detectors, some upload data to a central server where physicists or university students perform searches for coincidences among events at different locations. In other cases, the data upload is automatic. Currently, there are seven clusters with nearly 100 detection stations throughout the Netherlands.

\subsubsection{International Cosmic Day}

A newcomer in cosmic ray studies, International Cosmic Day (ICD) [13] led by physicists at DESY Zeuthen, is a program for schools that have access to a cosmic ray detector that can measure air showers or zenith angle distribution of air shower particles. Offered in fall, ICD is organized like a masterclass beginning with lectures, followed by running an experiment, making measurements, analyzing data, and presenting results on a poster. The day concludes with a videoconference where participants compare and discuss results. Some 31 sites participated in 2014. Organized by QuarkNet teachers, International Muon Week is a similar event offered in spring. [14]

\subsection{Research experiences}

Students in the United States have long summer vacations that provide time for researchers to offer eight- to ten-week internships where high school students can make a real contribution to ongoing research programs. QuarkNet has developed a program for teams composed of four students and one teacher who join a project for six weeks. Most effective is when the team is engaged in all aspects of research. Interns have an opportunity to discover the importance of, for example, troubleshooting, use of journals or logbooks, communicating ideas and results, and much more. Through these authentic research experiences, students have the greatest opportunity to gain a more sophisticated and complete understanding of scientific inquiry, to understand how scientists make discoveries and talk about their work. Students are unlikely to experience such learning in typical high school science classrooms.

\section{Conclusion}

We have presented a few examples of the many education and outreach activities that engage young people with our science. Developing an interest in science helps them begin an adventure that can lead to more challenging opportunities to learn about the excitement and value of cutting-edge scientific research. A few will become scientists, engineers, computing specialists or technicians. Others are the voters of tomorrow. Their willingness to support the research we are passionate about is crucial to the 
future of our field. It is not so difficult for members of our research community to participate in one of these activities, and many find it rewarding to work with these curious, interested young people.

\section{References}

1. International Particle Physics Outreach Group (IPPOG) online resources database: ippog.web.cern.ch/resources

2. Stockholm House of Science: http://www.vetenskapenshus.se/science for school

3. School Lab in DESY Zeuthen: http://physik-begreifen-zeuthen.desy.de/index eng.html

4. Fermilab's Saturday Morning Physics: https://indico.fnal.gov/conferenceDisplay.py?confId=4692

5. Saturday Physics for Everyone: http://physics.illinois.edu/outreach/saturdayphysics/

6. Cascades of Particle Physics: http://www.birmingham.ac.uk/schools/physics/outreach/activities/cascade-competitions.aspx

7. International Particle Physics Outreach Group (IPPOG): http://ippog.web.cern.ch

8. International Masterclasses: http://physicsmasterclasses.org

9. QuarkNet: https://quarknet.i2u2.org

10. e-Labs Website : https://www.i2u2.org/elab/

11. Cosmic Ray e-Lab: https://www.i2u2.org/elab/cosmic/home/project.jsp

12. High School Project on Astroparticle Research with Cosmics (HiSPARC): http://www.hisparc.nl/

13. International Cosmic Day (ICD: http://ippog.web.cern.ch/resources/2014/international-cosmic$\underline{\text { day-2014 }}$

14. International Muon Week: https://quarknet.i2u2.org/event/international-muon-week 